\title{
Existence and Uniqueness for Random One-Dimensional Lattice Systems
}

\author{
W. R. Schneider \\ Brown Boveri Research Center, $\mathrm{CH}-5405$ Baden, Switzerland
}

\begin{abstract}
Existence and uniqueness are shown for the fixed point problem pertinent to hopping transport in one-dimension with random transfer rates. Continuity properties of the solution are exhibited. The connection with Dyson's treatment of the linear harmonic chain with random masses is established.
\end{abstract}

\section{Introduction}

Diffusion or hopping transport on the one-dimensional lattice $\mathbb{Z}$ is described by the master equation

$$
\dot{P}_{n}=W_{n-1}\left(P_{n-1}-P_{n}\right)+W_{n}\left(P_{n+1}-P_{n}\right),
$$

where $P_{n}(t)$ is the probability of finding a particle at time $t$ on the lattice site $n$. Randomness is introduced by assuming the transfer rates $W_{n}, n \in \mathbb{Z}$, to be independent $\mathbb{R}_{+}$-valued random variables, equally distributed according to a probability measure $v$. Thus, one is lead to consider expectations

$$
E(f)=\int \prod_{n \in \mathbb{Z}} d v\left(w_{n}\right) f\left(\left\{w_{n}\right\}\right)
$$

of measurable functions $f$ on $\mathbb{R}_{+}^{\mathbb{Z}}$. In [1] it has been shown (by supplementing (1.1) with the initial condition $\left.P_{n}(0)=\delta_{n 0}\right)$, that

$$
E\left(\widetilde{P}_{0}(s)\right)=\int_{0}^{\infty} d t e^{-s t} E\left(P_{0}(t)\right)
$$

is given by

$$
E\left(\widetilde{P}_{0}(s)\right)=\iint_{\mathbb{R}_{+}^{2}} d \mu_{s}(x) d \mu_{s}(y)(x+y+s)^{-1}
$$

for $s \geqq 0$. Here, $\mu_{s}, s \in \mathbb{R}_{+}$, is a probability measure on $\mathbb{R}_{+}$satisfying the integral equation

$$
\mu_{s}([0, x))=\iint_{A_{s, x}} d v(y) d \mu_{s}(z), \quad x>0
$$


with $A_{\mathrm{s}, \mathrm{x}} \subset \mathbb{R}_{+}^{2}$ given by

$$
A_{s, x}=\left\{(y, z) \in \mathbb{R}_{+}^{2} \mid\left[y^{-1}+(z+s)^{-1}\right]^{-1}<x\right\} .
$$

In Sect. 2 it is shown that (1.5) has at most one solution. Section 3 is devoted to the existence of a solution; the solution is actually "constructed." In Sect. 4 it is shown that the map $s \rightarrow \mu_{s}$ is vaguely continuous. The connection with the work of Dyson [2] on the linear harmonic chain with random masses is established in Sect. 5. More detailed properties of $\mu_{s}$ and quantities derived thereof have been treated elsewhere [3], [4]; applications are discussed in [5].

\section{Uniqueness}

Let $\mathscr{P}$ be the set of (regular Borel) probability measures on $\mathbb{R}$, and $\mathscr{D}$ the set of distribution functions, i.e. the set of functions $f: \mathbb{R} \rightarrow[0,1]$ which are isotonic, leftcontinuous and $f(x)-f(-x) \rightarrow 1$ as $x \rightarrow \infty$. Denote by $J$ the canonical bijection of $\mathscr{P}$ onto $\mathscr{D}$ :

$$
(J \mu)(x)=\mu((-\infty, x)) .
$$

Let $\mathscr{P}_{+} \subset \mathscr{P}$ be the set of probability measures with support in $\mathbb{R}_{+}$and $\mathscr{D}_{+} \subset \mathscr{D}$ the set of distribution functions $f$ with $f(x)=0, x \leqq 0$. Obviously, $J$ maps $\mathscr{P}_{+}$ bijectively onto $\mathscr{D}_{+}$.

Let $v \in \mathscr{P}_{+}$be fixed and $s \in \mathbb{R}_{+} \cup\{\infty\}$. Define the map $T_{s}: \mathscr{P}_{+} \rightarrow \mathscr{P}_{+}$by

$$
\left(J T_{s} \mu\right)(x)=\iint_{A_{s, x}} d v(y) d \mu(z)
$$

with $A_{s, x} \subset \mathbb{R}_{+}^{2}$ given by (1.6). By definition, each fixed point of $T_{s}$ is a solution of the integral equation (1.5) and vice versa.

As

$$
A_{\infty, x}=[0, x) \times \mathbb{R}_{+},
$$

(2.2) yields immediately

$$
T_{\infty} \mu=v
$$

for all $\mu \in \mathscr{P}_{+}$, i.e. $T_{\infty}$ has the unique fixed point $\mu_{\infty}=v$. For $s \in \mathbb{R}_{+}$the following decomposition of $A_{s, x}$ into disjoint subsets holds

$$
A_{s, x}=A_{\infty, x} \cup B_{s, x} .
$$

The set $B_{s, x} \subset \mathbb{R}_{+}^{2}$ is given by

$$
B_{s, x}=\left\{(y, z) \mid z<\frac{y x}{y-x}-s, \quad x \leqq y<\phi_{s}(x)\right\},
$$

where

$$
\phi_{s}(x)= \begin{cases}s x /(s-x), & x<s \\ \infty & x \geqq s\end{cases}
$$


Obviously,

$$
B_{t, x} \varsubsetneqq B_{s, x}, \quad s<t .
$$

The decomposition (2.5) may easily be read off from the graph of $\psi_{s}: \mathbb{R}_{+}^{2} \rightarrow \mathbb{R}_{+}$ given by

$$
\psi_{s}(y, z)=\left[y^{-1}+(z+s)^{-1}\right]^{-1} .
$$

From (2.2), (2.5), (2.6) it follows that

$$
J T_{s} \mu=J \nu+K_{s} J \mu
$$

where

$$
\left(K_{s} J_{\mu}\right)(x)=\iint_{B_{s, x}} d v(y) d \mu(z)
$$

or, applying Fubini's theorem,

$$
\left(K_{s} f\right)(x)=\int_{x}^{\phi_{s}(x)} d v(y) f\left(\frac{y x}{y-x}-s\right)
$$

for $f \in \mathscr{D}_{+}$. Note that $K_{s}$ does not depend on $v(\{0\})$. In view of (2.8), the inequality

$$
K_{t} f \leqq K_{s} f, \quad s<t
$$

holds. The operator $K_{s}$ has an immediate extension from its "natural" domain $\mathscr{D}+$ to $D$, the linear span of $\mathscr{D}_{+}$. A further extension is obtained by introducing the Banach space

$$
\mathscr{B}_{\alpha}=L^{1}\left(\mathbb{R}_{+}, \rho_{\alpha}\right), \quad \frac{d \rho_{\alpha}}{d x}=\alpha(1+x)^{-1-\alpha}, \quad 0<\alpha<1,
$$

with norm

$$
\|f\|_{\alpha}=\alpha \int_{0}^{\infty} d x(1+x)^{-1-\alpha}|f(x)|
$$

Using Fubini's theorem and a change of variable

$$
x \rightarrow z=\frac{y x}{y-x}-s
$$

yields

$$
\left\|K_{s} f\right\|_{\alpha} \leqq \alpha \int_{0}^{\infty} d v(y) \int_{0}^{\infty} d z k_{s}(y, z)|f(z)|,
$$

with equality holding for $f \geqq 0$, and

$$
k_{s}(y, z)=y^{2}(s+y+z)^{-2}\left(1+\frac{y(s+z)}{s+y+z}\right)^{-1-\alpha}
$$


The estimates

$$
k_{s}(y, z)<\left(\frac{y}{y+s}\right)^{1-\alpha}(1+z)^{-1-\alpha} \leqq(1+z)^{-1-\alpha}
$$

which hold for $y>0, z>0$, lead to

$$
\left\|K_{s} f\right\|_{\alpha}<\|f\|_{\alpha} \int_{0}^{\infty} d v(y)\left(\frac{y}{y+s}\right)^{1-\alpha}, \quad f \neq 0,
$$

and

$$
\left\|K_{s} f\right\|_{\alpha}<\|f\|_{\alpha}, \quad f \neq 0 .
$$

By Lebesgue's dominated convergence theorem, (2.20) yields

$$
\lim _{s \rightarrow \infty}\left\|K_{s} f\right\|_{\alpha}=0,
$$

i.e. $K_{s}$ is strongly continuous at infinity.

As a consequence of (2.21), the equation

$$
f=g+K_{s} f
$$

has at most one solution $f \in \mathscr{B}_{\alpha}$ for any $g \in \mathscr{B}_{\alpha}, g \neq 0$. Thus, in view of (2.10), the following uniqueness theorem holds.

Theorem 2.1. The map $T_{s}: \mathscr{P}_{+} \rightarrow \mathscr{P}_{+}$, defined by (2.2), has at most one fixed point.

\section{Existence}

The functions

$$
f_{s}^{(n)}=\sum_{m=0}^{n} K_{s}^{m} J v, \quad n \geqq 0
$$

belong to $\mathscr{D}_{+}$, by induction, as

$$
f_{s}^{(n)}=J v+K_{s} f_{s}^{(n-1)},
$$

and, from (2.10), with $f_{s}^{(n-1)} \in \mathscr{D}_{+}$also

$$
f_{\mathrm{s}}^{(n)}=J T_{\mathrm{s}} J^{-1} f_{\mathrm{s}}^{(n-1)}
$$

is in $\mathscr{D}_{+}$. Furthermore, (3.1) yields

$$
f_{s}^{(n)}=f_{s}^{(n-1)}+K_{s}^{n} J v .
$$

Hence,

$$
0 \leqq f_{s}^{(0)} \leqq f_{s}^{(1)} \leqq \ldots \leqq f_{s}^{(n)} \leqq \ldots \leqq 1,
$$

as $K_{s}$ is positivity preserving. Consequently,

$$
\lim _{n \rightarrow \infty} f_{s}^{(n)}=f_{s}
$$


exists pointwise. As each $f_{s}^{(n)}, n \geqq 0$, is isotonic, also $f_{s}$ is isotonic. Thus, the limits

$$
\lim _{y \uparrow x} f_{s}(y)=f_{s}\left(x_{-}\right), \quad \lim _{y \downarrow x} f_{s}(y)=f_{s}\left(x_{+}\right)
$$

exist. Assume $f_{s}$ not to be left-continuous, i.e.

$$
f_{s}(x)-f_{s}\left(x_{-}\right)=a>0 .
$$

For $n$ sufficiently large (say $n>N$ )

$$
0 \leqq f_{s}(x)-f_{s}^{(n)}(x)<a / 2, \quad n>N,
$$

and for $y<x$

$$
0 \leqq f_{s}^{(n)}(y) \leqq f_{s}(y) \leqq f_{s}\left(x_{-}\right)
$$

i.e.

$$
f_{s}^{(n)}(x)-f_{s}^{(n)}(y)>a / 2, \quad n>N .
$$

Taking the limit $y \uparrow x$ yields

$$
f_{s}^{(n)}(x)-f_{s}^{(n)}\left(x_{-}\right) \geqq a / 2, \quad n>N .
$$

This contradicts the left-continuity of $f_{s}^{(n)}, n \geqq 0$. Finally (3.5) yields

$$
\lim _{x \rightarrow \infty} f_{s}(x)=1 \text {, }
$$

and

$$
f_{s}^{(n)}=0, \quad x \leqq 0, \quad n \geqq 1
$$

yields

Hence, $f$ is in $\mathscr{D}_{+}$.

$$
f_{s}(x)=0, \quad x \leqq 0
$$

Furthermore, by Lebesgue's dominated convergence theorem

$$
\lim _{n \rightarrow \infty}\left\|f_{s}-f_{s}^{(n)}\right\|_{\alpha}=0 .
$$

As $K_{s}$ is bounded, (3.2) combined with (3.16) leads to

$$
f_{s}=J v+K_{s} f_{s}
$$

or, with (2.10)

$$
f_{s}=J T_{s} J^{-1} f_{s}
$$

Hence, the following theorem holds.

Theorem 3.1. The sequence

$$
J T_{s}^{n} v=\sum_{m=0}^{n} K_{s}^{m} J v
$$

is in $\mathscr{D}_{+}$. It converges pointwise and in $\mathscr{B}_{\alpha}$-norm. Its limit, $f_{s}$, defines a probability measure $\mu_{s}=J^{-1} f_{s}$ which is a fixed point of $T_{s}$. 
Remark 1. As $K_{\infty}=0,(3.1)$ reduces to $f_{\infty}^{(n)}=J v, n \geqq 1$, i.e. $f_{\infty}=J v$, in accordance with Sect. 2.

Remark 2. Let $f_{0} \in \mathscr{D}_{+}$be given by

$$
f_{0}(x)=\left\{\begin{array}{ll}
0, & x \leqq 0 \\
1, & x>0
\end{array} .\right.
$$

Applying $K_{0}$ to $f_{0}$ yields, according to (2.12) and (2.7),

$$
K_{0} f_{0}=f_{0}-J v
$$

i.e. $J^{-1} f_{0}=\delta_{0}$ (Dirac measure) is fixed point of $T_{0}$.

Remark 3. The ordered case of (1.1) is characterized by $W_{n}=w, w \geqq 0, n \in \mathbb{Z}$. This is equivalent to $v=\delta_{w}$. For $w=0,(2.2)$ yields

$$
J T_{s} \mu=f_{0}
$$

for arbitrary $\mu \in \mathscr{P}_{+}$, i.e. $\delta_{0}$ is fixed point of $T_{s}, s \in \mathbb{R}_{+} \cup\{\infty\}$. For $w>0$, the point $(w, a(s))$ with

$$
a(s)=\frac{1}{2}\left[\left(4 w s+s^{2}\right)^{1 / 2}-s\right], \quad s \in \mathbb{R}_{+},
$$

and

$$
a(\infty)=\lim _{s \rightarrow \infty} a(s)=w
$$

is mapped onto itself by $\psi_{s}$ defined in (2.8). Hence, $\mu_{s}=\delta_{a(s)}$ is fixed point of $T_{s}$, $s \in \mathbb{R}_{+} \cup\{\infty\}$.

Remark 4. Replacing $A_{s, x}$ in (2.2) by its closure and taking the limit $x \rightarrow 0$ yields $\left(T_{s} \mu\right)(\{0\})=v(\{0\})$. In particular,

$$
\mu_{s}(\{0\})=v(\{0\}) .
$$

\section{Continuity Properties}

In this section continuity properties of $K_{s}$ and $\mu_{s}$ are discussed. Let $\mathscr{C}\left(\mathscr{B}_{\alpha}\right)$ be the set of bounded operators on $\mathscr{B}_{\alpha}$.

Theorem 4.1. The map $s \rightarrow K_{s}$ from $\mathbb{R}_{+} \cup\{\infty\}$ to $\mathscr{C}\left(\mathscr{B}_{\alpha}\right)$, defined by (2.10) and (2.7), is strongly continuous.

Proof. As $K_{\infty}=0$, strong continuity at $\infty$ is equivalent to (2.22). Let $0 \leqq s<t<\infty$ and $f \in \mathscr{B}_{\alpha}, f \geqq 0$. Then

$$
\left\|K_{t} f-K_{s} f\right\|_{\alpha}=\alpha \int_{0}^{\infty} d v(y) \int_{0}^{\infty} d z\left[k_{s}(y, z)-k_{t}(y, z)\right] f(z)
$$

with $k_{s}$ given by (2.18). In view of the estimates (2.19) Lebesgue's dominated 
convergence theorem is applicable yielding

$$
\lim _{s \rightarrow t}\left\|K_{t} f-K_{s} f\right\|_{\alpha}=0 .
$$

The extension to arbitrary $f \in \mathscr{B}_{\alpha}$ is trivial as $K_{s}$ is positivity preserving.

Theorem 4.2. The map

$$
s \rightarrow f_{s}=\sum_{m=0}^{\infty} K_{s}^{m} J v
$$

from $\mathbb{R}_{+} \cup\{\infty\}$ to $\mathscr{B}_{\alpha}$ is continuous.

Proof. By Theorem 4.1, $K_{s}^{m} J v$ is continuous. Hence, $f_{s}^{(n)}, n \geqq 1$, given by (3.1), is continuous. From

$$
f_{s}^{(n)} \leqq f_{0}^{(n)}, n \geqq 1,
$$

shown below by induction, it follows that $f_{s}^{(n)}$ converges uniformly to $f_{s}$. Hence, $f_{s}$ is continuous. For $n=1,(3.2)$ and (2.13) yield

$$
f_{s}^{(1)}=J v+K_{s} J v \leqq J v+K_{0} J v=f_{0}^{(1)} .
$$

Assume $f_{s}^{(n-1)} \leqq f_{0}^{(n-1)}$. Again using (3.2) and (2.13) leads to

$$
f_{s}^{(n)}=J v+K_{s} f_{s}^{(n-1)} \leqq J v+K_{0} f_{s}^{(n-1)} \leqq J v+K_{0} f_{0}^{(n-1)}=f_{0}^{(n)} .
$$

This completes the proof.

Let $C_{0}(\mathbb{R})$ denote the set of $\mathbb{R}$-valued continuous functions on $\mathbb{R}$ with compact support, and $C_{0}^{1}(\mathbb{R})$ the subset consisting of the functions in $C_{0}(\mathbb{R})$ having a continuous derivative.

Theorem 4.3. The map $s \rightarrow \mu_{s}=J^{-1} f_{s}$ from $\mathbb{R}_{+} \cup\{\infty\}$ to $\mathscr{P}_{+}$is vaguely continuous, i.e.

$$
\mu_{s}(g)=\int g(x) d \mu_{s}(x), \quad g \in C_{0}(\mathbb{R})
$$

depends continuously on $s$.

Proof. It is sufficient to prove the latter statement for $h \in C_{0}^{1}(\mathbb{R})$ as $C_{0}^{1}(\mathbb{R})$ is dense in $C_{0}(\mathbb{R})$ with respect to the sup-norm. Partial integration leads to

$$
\mu_{s}(h)=-\int h^{\prime}(x) f_{s}(x) d x,
$$

where $h^{\prime}$ is the derivative of $h$. Hence,

$$
\left|\mu_{t}(h)-\mu_{s}(h)\right| \leqq \int\left|h^{\prime}(x)\right|\left|f_{t}(x)-f_{s}(x)\right| d x .
$$

Setting

$$
C_{\alpha}(h)=\sup _{x \in \mathbb{R}_{+}}\left|h^{\prime}(x)\right| \rho_{\alpha}(x)^{-1},
$$

(4.9) yields the estimate

$$
\left|\mu_{t}(h)-\mu_{s}(h)\right| \leqq C_{\alpha}(h)\left\|f_{t}-f_{s}\right\|_{\alpha},
$$

which, together with Theorem 4.2., proves continuity of $\mu_{s}(h)$ in $s$. 


\section{The Disordered Harmonic Chain}

A fixed point problem similar to the one of Sect. 1 was posed by Dyson [2] in the context of the mass-disordered infinite linear harmonic chain. Results analogous to those of Sects. 2-4 are obtained, and the connection between the two fixed point problems is exhibited.

An infinite linear harmonic chain is described by the equations of motion

$$
M_{n} \ddot{Q}_{n}=W_{n-1}\left(Q_{n-1}-Q_{n}\right)+W_{n}\left(Q_{n+1}-Q_{n}\right), \quad n \in \mathbb{Z} .
$$

Here $M_{n}$ is the mass of the $n^{\text {th }}$ particle, $Q_{n}$ its displacement from its equilibrium position and $W_{n}$ the spring constant of the spring between particle $n$ and $n+1$.

Several variants of disorder may be envisaged, involving randomness of masses and spring constants. The case considered here is case II of Dyson, where $M_{n}, n \in \mathbb{Z}$, are independent equally distributed $\mathbb{R}_{+}$-valued random variables, whereas the spring constants $W_{n}$ have a common fixed value. Let $\tau \in \mathscr{P}+$ denote the probability measure describing the distribution of the masses. It is assumed that

$$
\tau(\{0\})=0
$$

i.e. there are no zero-mass particles, or more stringent,

$$
\tau([0, m))=0, \quad m>0
$$

i.e. a mass gap.

Dyson's fixed point problem consists in finding a probability measure $\rho_{s} \in \mathscr{P}_{+}$ satisfying

$$
\rho_{s}=R_{s} \rho_{s}, \quad s \in \mathbb{R}_{+} .
$$

The map $R_{s}: \mathscr{P}_{+} \rightarrow \mathscr{P}_{+}$is given by

$$
\left(J R_{s} \rho\right)(x)=\iint_{C_{s, x}} d \tau(y) d \rho(z)
$$

with

$$
C_{s, x}=\left\{(y, z) \in \mathbb{R}_{+}^{2} \mid s y+z /(1+z)<x\right\} .
$$

From (5.6) it follows that (5.5) may be rewritten as

$$
J R_{s} \rho=J \tau_{s}+H_{s} J \rho .
$$

For $s>0$ the two parts of (5.7) are given by

$$
\left(J \tau_{s}\right)(x)=(J \tau)\left(\frac{x-1}{s}\right),
$$

and, with $f \in \mathscr{D}_{+}$,

$$
\left(H_{s} f\right)(x)=\int_{\beta_{s}(x)}^{x / s} d \tau(y) f\left(\frac{x-s y}{1-x+s y}\right)
$$


where

$$
\beta_{s}(x)=\max \left\{0, \frac{x-1}{s}\right\} .
$$

The case $s=0$ is obtained either directly from (5.5), (5.6), i.e. from

$$
\left(J R_{0} \rho\right)(x)= \begin{cases}J \rho\left(\frac{x}{1-x}\right), & x<1, \\ 1, & x \geqq 1,\end{cases}
$$

or as limits from (5.8) and (5.9), yielding

$$
\left(J \tau_{0}\right)(x)= \begin{cases}0, & x \leqq 1 \\ 1, & x>1\end{cases}
$$

and

$$
\left(H_{0} f\right)(x)= \begin{cases}f\left(\frac{x}{1-x}\right), & x<1 \\ 0, & x>1\end{cases}
$$

supplemented by

$$
\left(H_{0} f\right)(1)=\lim _{x \uparrow 1} f\left(\frac{x}{1-x}\right) \quad\left(=1 \text { for } f \in \mathscr{D}_{+}\right) .
$$

Extension of (5.9), (5.13) and (5.14) to $f \in D$, the linear span of $\mathscr{D}_{+}$, is immediate. A further extension of (5.9) and (5.13) to the Banach space $\mathscr{B}_{\alpha}$, defined in (2.14), leads to the estimate (equality holding for $f \geqq 0$ )

$$
\left\|H_{s} f\right\|_{\alpha} \leqq \alpha \int_{0}^{\infty} d \tau(y) \int_{0}^{\infty} d z h_{s}(y, z)|f(z)|, \quad s \in \mathbb{R}_{+},
$$

with

$$
h_{s}(y, z)=(1+z)^{-2}\left(1+\frac{z}{1+z}+s y\right)^{-1-\alpha} .
$$

The inequalities

$$
h_{s}(y, z)<(1+z)^{-1-\alpha}(1+s y)^{-1-\alpha}<(1+z)^{-1-\alpha},
$$

holding for $y>0, z>0$, imply for $f \neq 0$

$$
\left\|H_{s} f\right\|_{\alpha}<\|f\|_{\alpha} \int^{\infty} d \tau(y)(1+s y)^{-1-\alpha}<\|f\|_{\alpha} .
$$

This yields uniqueness for $f \in \mathscr{B}_{\alpha}$, satisfying $f=g+H_{s} f, g \in \mathscr{B}_{\alpha}, g \neq 0$. In particular, there is at most one solution of (5.4). For $s=0$, there is a solution, namely

as may be verified with (5.11).

$$
\rho_{0}=\delta_{0},
$$


Existence of a solution for $s>0$ is obtained by introducing the sequence

$$
g_{\mathrm{s}}^{(n)}=\sum_{m=0}^{n} H_{s}^{m} J \tau_{s}=J R_{\mathrm{s}}^{n} \tau_{s},
$$

$n=0,1,2, \ldots$ As in Sect. 3 one shows that $g_{s}^{(n)} \rightarrow g_{s} \in \mathscr{D}_{+}$pointwise, and in $\mathscr{B}_{\alpha}$, as $n \rightarrow \infty$, with $g_{s}$ satisfying $g_{s}=J R_{s} J^{-1} g_{s}$, i.e. $\rho_{s}=J^{-1} g_{s}$ is fixed point of $R_{s}$.

For the ordered case $\tau=\delta_{m}$, the solution is given by

$$
\rho_{s}=\delta_{b(s)}, \quad b(s)=\frac{1}{2}\left\{m s+\left(4 m s+m^{2} s^{2}\right)^{1 / 2}\right\} .
$$

As in Sect. 4 one show that $s \rightarrow g_{s}$ is $\mathscr{B}_{\alpha}$-continuous and $s \rightarrow \rho_{s}$ is vaguely continuous. There is, however, a difference in behaviour of $f_{s}$ and $g_{s}$ with respect to the limit $s \rightarrow \infty$. The former satisfies

$$
\lim _{s \rightarrow \infty} f_{s}=f_{\infty}=J v \text { in } \mathscr{B}_{\alpha}
$$

the latter

$$
\lim _{s \rightarrow \infty} g_{s}=0 \text { in } \mathscr{B}_{\alpha} .
$$

At a first glance, the two fixed point problems $\mu_{s}=T_{s} \mu_{s}$ and $\rho_{s}=R_{s} \rho_{s}$ of Sects. 1 and 5 , respectively, seem to be similar only with respect to their general structure, but there is a deeper relationship. Actually, $\rho_{s}$ may be obtained from $\mu_{s}$ by choosing $v$ appropriately.

Set $f(x)=0$ for $x \leqq 0$ and

$$
f(x)=\tau\left(\left(x^{-1}, \infty\right)\right), \quad x>0
$$

One verifies easily $f \in \mathscr{D}_{+}$, taking (5.2) into account. Hence,

$$
J^{-1} f=v \in \mathscr{P}_{+}
$$

and

$$
v(\{0\})=\lim _{x \downarrow 0} f(x)=\lim _{x \downarrow 0}\left\{1-\tau\left(\left[0, x^{-1}\right]\right\}=0 .\right.
$$

Solving $\mu_{s}=T_{s} \mu_{s}$, with $v$ given by (5.24), (5.25) yields $\mu_{s} \in \mathscr{P}_{+}$, which satisfies $\mu_{s}(\{0\})=0$ in view of (5.26) and (3.25). This implies that $g_{s}$ with $g_{s}(x)=0$ for $x \leqq 0$ and

$$
g_{s}(x)=\mu_{s}\left(\left(s x^{-1}, \infty\right)\right)
$$

is in $\mathscr{D}_{+}$. It satisfies $J R_{s} J^{-1} g_{s}=g_{s}$, as shown below, i.e. $\rho_{s}=J^{-1} g_{s} \in \mathscr{P}_{+}$is fixed point of $R_{s}$. Now,

$$
\begin{aligned}
g_{s}(x) & =\mu_{s}\left(\left(s x^{-1}, \infty\right)\right)=\left(T_{s} \mu_{s}\right)\left(\left(s x^{-1}, \infty\right)\right) \\
& =\int_{\mathbb{R}_{+}^{2} \backslash \bar{A}_{s, s x-1}} d v(u) d \mu_{s}(v),
\end{aligned}
$$

with $\bar{A}$ being the closure of $A$.

Now, for $u \neq 0$ and $v \neq 0$,

$$
(u, v) \in \mathbb{R}_{+}^{2} \backslash \bar{A}_{s, s x-1} \Leftrightarrow\left(u^{-1}, s v^{-1}\right) \in C_{s, x} .
$$


Hence, in view of (5.24) and (5.27),

$$
g_{s}(x)=\iint_{C_{s, x}} d \tau(y) d \rho_{s}(z)
$$

which proves the invariance of $\rho_{s}=J^{-1} g_{s}$.

\section{References}

1. Bernasconi, J., Alexander, S., Orbach, R.: Classical diffusion in one-dimensional disordered lattice. Phys. Rev. Lett. 41, 185-187 (1978)

2. Dyson, F. J.: The dynamics of a disordered linear chain. Phys. Rev. 92, 1331-1338 (1953).

3. Bernasconi, J., Schneider, W. R., Wyss, W.: Diffusion and hopping conductivity in disordered onedimensional lattice systems. Z. Phys. B37, 175-184 (1980)

4. Schneider, W. R., Bernasconi, J.: In: Lecture Notes in Physics, Vol. 153, pp. 389-393. Berlin, Heidelberg, New York: Springer 1982.

5. Alexander, S., Bernasconi, J., Schneider, W. R., Orbach, R.: Excitation dynamics in randóm onedimensional systems. Rev. Mod. Phys. 53, 175-198 (1981)

Communicated by Ya. G. Sinai

Received January 10, 1982 
